\title{
Factor de impacto agregado según campos científicos
}

\author{
María Isabel Dorta González * \\ Pablo Dorta González **
}

Artículo recibido: 20 de septiembre de 2012.

Artículo aceptado:

7 de agosto de 2013.

\section{Resumen}

Los indicadores de impacto de revistas no son comparables entre campos científicos debido a las diferencias significativas en los hábitos de publicación y citación. Una descomposición del factor de impacto agregado muestra, para los 22 campos y cuatro áreas consideradas en las bases de datos del principal proveedor de indicadores científicos (Thomson Reuters), que existen cinco variables que en mayor medida explican la varianza del factor de impacto de un campo. Por ello es necesario tener en cuenta estas fuentes de variación en el proceso de normalización de los indicadores de impacto. Para localizar las fuentes de la varianza se emplea un Análisis de componentes principales y para detectar las semejanzas se utiliza un Análisis clúster.

* Universidad de La Laguna, Tenerife, España. isadorta@ull.es

** Universidad de Las Palmas de Gran Canaria, España. pdorta@dmc.ulpgc.es

INVESTIGACIÓN BIBLIOTECOLÓGICA, Vol. 28, Núm.62, enero/abril, 2014, México, ISSN 0187-358X. pp. 15-28 
Palabras clave: Factor de Impacto; Evaluación de Revistas; Análisis de Componentes Principales; Análisis Clúster.

\section{Abstract}

Aggregate Impact Factor According to Scientific Fields

María-Isabel Dorta-González and Pablo Dorta-González

Science journal impact indicators are not comparable because of inherent differences in publication and citation behaviors from field to field. A breakdown of the field aggregate impact factor of databases shows that for the 22 fields and four areas considered by Thomson Reuters, the leading provider of science indicators, five variables largely explain variance in impact factor of a given field. Therefore, it is necessary to consider all these sources of variance in the standardization process of the impact indicators. A Principal Component Analysis is employed to find the sources of the variance and a Cluster Analysis is used to detect similarities.

Keywords: Impact Factor; Journal Evaluation; Principal Component Analysis; Cluster Analysis.

\section{INTRODUCCIÓN}

$\mathrm{C}^{1}$ Factor de Impacto (FI) publicado en el Journal Citation Reports (JCR) $\mathrm{C}_{\text {por Thomson Reuters se define como el número de las referencias pro- }}$ medio que recibe una revista en un año determinado de los 'ítems citables' publicados en esa revista durante los dos años previos. Desde su presentación (Garfield, 1972), el FI ha sido criticado por algunas decisiones arbitrarias en su formulación. En la literatura se han discutido aspectos como la definición de ítems citables (artículos, revisiones, actas de congresos y cartas), y el hecho de centrarse en los dos años precedentes como parte representativa del frente de investigación, etc. (Bensman, 2007), y han sido sugeridas numerosas modificaciones (Althouse et al. 2009; Bornmann y Daniel, 2008; Dorta-González y Dorta-González, 2013a,b). 
El origen del problema de comparar revistas de diferentes campos científicos proviene de la evaluación institucional (Leydesdorff y Opthof, 2010; Opthof y Leydesdorff, 2010; Van Raan et al., 2010). La distribución de citas varía según los campos científicos y, en algunos casos, dentro de las propias especialidades de los campos (Dorta-González y Dorta-González, 2010, 2011a,b). Sin embargo, los centros de investigación están integrados por investigadores de disciplinas muy diversas y tienen con frecuencia entre sus misiones el objetivo de integrar grupos multidisciplinares (Leydesdorff y Rafols, 2011; Wagner et al., 2011).

La mayoría de los trabajos sobre clasificación de revistas en campos científicos se han centrado en la correlación entre los patrones de citación (Leydesdorff, 2006; Rosvall y Bergstrom, 2008). Índices como el JCR Subject Category List clasifican las revistas en diferentes grupos (Pudovkin y Garfield, 2002; Rafols y Leydesdorff, 2009). En este sentido, Egghe y Rousseau (2002) definen el Relative Impact Factor de forma similar al FI, agregando todas las revistas de una categoría como una única meta-revista. Este indicador se denomina Aggregate Impact Factor en el JCR.

Existen algunos patrones estadísticos propios de los campos. Garfield (1979a,b) propone el término potencial de citación, con base en el número de referencias promedio, para justificar las diferencias sistemáticas entre campos científicos. Por ejemplo, en biomedicina son comunes los listados con más de 50 referencias, mientras que en matemáticas lo frecuente es incluir menos de 20 referencias. Estas diferencias se deben a las distintas culturas de citación y afectan significativamente al FI debido a que condicionan la probabilidad de ser citado. El recuento fraccional corrige estas diferencias basándose en las fuentes de las citas (Leydesdorff y Bornmann, 2011; Moed, 2010; Zitt y Small, 2008). Así, una cita de un artículo citante que contiene $n$ referencias cuenta $1 / n$ en el caso del recuento fraccional, mientras que vale 1 en el caso del recuento entero.

Otra fuente importante de varianza entre los campos es el canal de difusión que muestra los resultados de la actividad investigadora. Por ejemplo, los investigadores de ciencias sociales y humanidades publican más en libros que en revistas, y los investigadores de ciencias de la computación publican más en actas de congresos que en artículos de revistas. Las diferencias entre los campos se deben principalmente a la proporción de referencias del JCR frente a otras como libros, revistas no incluidas en el JCR, etc. (Althouse et al., 2009).

El objetivo de este trabajo es identificar las fuentes de varianza de acuerdo con los campos científicos. La identificación de estas fuentes es fundamental a la hora de implementar indicadores bibliométricos normalizados y 
ajustados a las particularidades de cada campo. Además de las variables estudiadas en la literatura (el número de referencias promedio y la proporción de referencias en el JCR), en este trabajo se consideran tres nuevas variables: el índice de crecimiento del campo, la proporción de referencias del JCR en la ventana de citas y la proporción entre los ítems citados y los citantes. Se presenta una descomposición del factor de impacto en estas cinco fuentes principales de varianza para todos los campos y áreas científicas considerados en las bases de datos de Thomson Reuters.

\section{DESCOMPOSICIÓN DEL FACTOR DE IMPACTO EN SUS COMPONENTES}

El impacto de una revista es una medida del número de veces que los artículos de cierto periodo censal citan artículos publicados durante una ventana de citación previa. El Factor de Impacto (FI) de una revista proporcionado por Thomson Reuters utiliza un periodo censal de un año y una ventana de citación de dos años.

El Factor de Impacto Agregado (FIA) de un campo se obtiene al considerar todas las revistas de dicho campo como un todo. Como un promedio, el cálculo del FIA depende de dos elementos: el numerador es el número de citas en el año actual sobre cualquiera de los ítems publicados en las revistas del campo en los dos años previos, y el denominador es el número de ítems citables publicados en esos dos años. Los ítems publicados incluyen los ítems citables (artículos, revisiones, actas de congresos y cartas), pero también correcciones, editoriales y noticias, entre otros.

Es factible comprobar que el factor de impacto agregado del campo $F$ en el año $t$ se puede descomponer de la siguiente manera:

$$
F I A_{t}^{F}=a_{t}^{F} \cdot r_{t}^{F} \cdot p_{t}^{F} \cdot w_{t}^{F} \cdot b_{t}^{F}
$$

donde $a_{t}^{F}$ es un indicador del crecimiento del campo y $r_{t}^{F} \cdot p_{t}^{F} \cdot w_{t}^{F} \cdot b_{t}^{F}$, son cuatro indicadores del hábito de citación en dicho campo. Estas variables se definen en la Tabla 1.

Tabla 1. Variables empleadas en la descomposición del factor de impacto.

\begin{tabular}{|l|l|l|}
\hline Notación & \multicolumn{1}{|c|}{ Definición } & \multicolumn{1}{c|}{ Descripción } \\
\hline$a_{t}^{F}$ & Ratio de crecimiento. & $\begin{array}{l}\text { Cociente entre los ítems citables en el año ty aquellos que } \\
\text { aparecen en la ventana de citación. }\end{array}$ \\
\hline$r_{t}^{F}$ & Número de referencias promedio. & $\begin{array}{l}\text { Cociente entre el número total de referencias y el número } \\
\text { total de ítems citables. }\end{array}$ \\
\hline
\end{tabular}




\begin{tabular}{|l|l|l|}
\hline$p_{t}^{F}$ & Proporción de referencias del JCR. & $\begin{array}{l}\text { Cociente entre el número total de referencias a revistas del } \\
J C R \text { (excluidos documentos de trabajo, actas, libros y re- } \\
\text { vistas no indexadas) y el total de referencias. }\end{array}$ \\
\hline$w_{t}^{F}$ & $\begin{array}{l}\text { Proporción de referencias del } J C R \\
\text { en la ventana de citación. }\end{array}$ & $\begin{array}{l}\text { Cociente entre el total de referencias del } J C R \text { en la ventana } \\
\text { de citación y el total de referencias del propio } J C R .\end{array}$ \\
\hline$b_{t}^{F}$ & $\begin{array}{l}\text { Proporción entre ítems citados y y } \\
\text { citantes en la ventana de citación. }\end{array}$ & $\begin{array}{l}\text { Cociente entre el total de citas recibidas y el de citas reali- } \\
\text { zadas dentro de la ventana de citación. }\end{array}$ \\
\hline
\end{tabular}

El crecimiento de un campo se puede deber a dos razones principales, la incorporación de nuevas revistas y la publicación de ítems adicionales en las revistas ya incluidas. No obstante, un campo también puede decrecer. Téngase en cuenta que $a_{t}^{F}=0.5$ cuando coincide el número de ítems citables en los años $t, t-1, t-2$. Si $a_{t}^{F}>0.5$, se produce un crecimiento del campo con respecto al número de ítems citables. En otro caso, $a_{t}^{F}>0.5$, se produce un decrecimiento.

Aunque la mayoría de las citas a una revista proceden de revistas del mismo campo, algunas otras provienen de revistas de otros campos. Cuando $b_{t}^{F}>1$, las citas recibidas por el campo $F$ son mayores que las producidas en ese campo. En otro caso, $b_{t}^{F}<1$, las citas recibidas en el campo $F$ son menores que las producidas en ese campo. Este indicador es, por tanto, una medida del intercambio de citas entre campos. Por ejemplo, si $b_{t}^{F}=1.1$, el campo $F$ recibe un $10 \%$ más de citas de las que produce.

El resto de las variables son proporciones. Por ejemplo, si $p_{t}^{F}=0.5$, la mitad de las referencias son ítems JCR, y si $w_{t}^{F}=0.25$, una cuarta parte de las referencias JCR pertenecen a la ventana de citación.

\section{MATERIALES Y MÉTODOS}

Los datos bibliométricos se obtuvieron de la versión en línea del Journal $\mathrm{C} i$ tation Reports (JCR) durante la primera semana de octubre de 2011 ( $t=2010)$. La base de datos del JCR (proporcionada por Thomson Reuters, Philadelphia, USA) está disponible en la dirección www.webofknowledge.com.

Thomson Reuters asigna cada revista JCR a una o más categorías de acuerdo con sus revistas citantes y citadas (Pudovkin y Garfield, 2002). La edición de Ciencias 2010 contiene 8073 revistas clasificadas en 174 categorías, y la edición de Ciencias Sociales 2010 contiene 2731 revistas clasificadas en 56 categorías.

En este trabajo se consideran dos niveles de agregación de datos. El primer nivel se corresponde con los 22 campos cientificos y el segundo con las cuatro áreas científicas, ambos considerados en las bases de datos de Thomson Reuters. 
Las variables empleadas se describen en la Tabla 1. Respecto a los métodos utilizados, para localizar las fuentes de la varianza se emplea un Análisis de Componentes Principales y para detectar las semejanzas se utiliza un Análisis Clúster.

\section{RESULTADOS Y DISCUSIÓN}

La Tabla 2 muestra el Impacto Agregado y las componentes para los campos científicos de las bases de datos de Thomson Reuters. Las categorías de las revistas del JCR que constituyen cada campo aparecen en el Anexo 1 junto al FIA de la categoría y al porcentaje obtenido dentro del campo. El FIA de Ciencias es 2.920, un 58 \% mayor que el de Ciencias Sociales (1.848).

Tabla 2. Factor de impacto agregado y componentes para los campos científicos de las bases de datos de Thomson Reuters.

\begin{tabular}{|c|c|c|c|c|c|c|c|c|}
\hline \multirow[t]{2}{*}{ Código } & \multirow[t]{2}{*}{ Campo } & \multirow{2}{*}{$\begin{array}{l}\text { Núm. } \\
\text { categorías }\end{array}$} & \multicolumn{6}{|c|}{ Componentes del FIA } \\
\hline & & & $a_{t}^{F}$ & $r_{t}^{F}$ & $p_{t}^{F}$ & $w_{t}^{F}$ & $b_{t}^{F}$ & $F I A_{t}^{F}$ \\
\hline C1 & $\begin{array}{l}\text { Agricultural } \\
\text { Sciences }\end{array}$ & 6 & 0.58 & 35.93 & 0.80 & 0.16 & 0.80 & 2.142 \\
\hline $\mathrm{C} 2$ & $\begin{array}{l}\text { Biology \& Bioche- } \\
\text { mistry }\end{array}$ & 12 & 0.56 & 45.86 & 0.90 & 0.18 & 0.92 & 3.859 \\
\hline C3 & Clinical Medicine & 50 & 0.56 & 38.84 & 0.87 & 0.19 & 0.92 & 3.330 \\
\hline C4 & $\begin{array}{l}\text { Computer } \\
\text { Science }\end{array}$ & 9 & 0.54 & 30.22 & 0.63 & 0.21 & 0.72 & 1.529 \\
\hline $\mathrm{C5}$ & Chemistry & 15 & 0.55 & 37.20 & 0.90 & 0.19 & 0.87 & 3.061 \\
\hline C6 & $\begin{array}{l}\text { Economics \& } \\
\text { Business }\end{array}$ & 8 & 0.62 & 45.82 & 0.66 & 0.15 & 0.59 & 1.642 \\
\hline $\mathrm{C7}$ & Engineering & 39 & 0.55 & 27.74 & 0.77 & 0.19 & 0.84 & 1.931 \\
\hline $\mathrm{C} 8$ & $\begin{array}{l}\text { Environment/ } \\
\text { Ecology }\end{array}$ & 8 & 0.54 & 44.55 & 0.76 & 0.19 & 0.75 & 2.569 \\
\hline C9 & Geosciences & 13 & 0.55 & 42.96 & 0.79 & 0.15 & 0.77 & 2.232 \\
\hline C10 & Immunology & 2 & 0.52 & 42.66 & 0.90 & 0.22 & 1.01 & 4.342 \\
\hline C11 & $\begin{array}{l}\text { Materials } \\
\text { Science }\end{array}$ & 11 & 0.55 & 30.80 & 0.88 & 0.20 & 0.91 & 2.714 \\
\hline C12 & Mathematics & 6 & 0.55 & 25.75 & 0.77 & 0.15 & 0.82 & 1.345 \\
\hline C13 & Microbiology & 5 & 0.56 & 43.25 & 0.90 & 0.19 & 0.91 & 3.638 \\
\hline C14 & $\begin{array}{l}\text { Molecular Biology } \\
\& \text { Genetics }\end{array}$ & 5 & 0.53 & 51.64 & 0.92 & 0.19 & 1.06 & 5.083 \\
\hline C15 & Multidisciplinary & 1 & 0.58 & 36.81 & 0.84 & 0.21 & 2.55 & 9.747 \\
\hline C16 & $\begin{array}{l}\text { Neuroscience \& } \\
\text { Behavior }\end{array}$ & 5 & 0.54 & 49.19 & 0.90 & 0.16 & 0.95 & 3.653 \\
\hline
\end{tabular}




\begin{tabular}{|c|l|c|c|c|c|c|c|c|}
\hline C17 & $\begin{array}{l}\text { Pharmacology \& } \\
\text { Toxicology }\end{array}$ & 3 & 0.55 & 46.16 & 0.87 & 0.20 & 0.69 & 3.013 \\
\hline C18 & Physics & 11 & 0.52 & 30.21 & 0.90 & 0.19 & 0.97 & 2.617 \\
\hline C19 & $\begin{array}{l}\text { Plant \& Animal } \\
\text { Science }\end{array}$ & 15 & 0.54 & 43.27 & 0.81 & 0.14 & 0.75 & 1.980 \\
\hline C20 & $\begin{array}{l}\text { Psychiatry/Psy- } \\
\text { chology }\end{array}$ & 17 & 0.55 & 50.28 & 0.79 & 0.15 & 0.83 & 2.663 \\
\hline C21 & $\begin{array}{l}\text { Social Sciences, } \\
\text { general }\end{array}$ & 51 & 0.63 & 44.33 & 0.61 & 0.21 & 0.49 & 1.736 \\
\hline C22 & Space Science & 1 & 0.47 & 56.59 & 0.78 & 0.24 & 0.92 & 4.621 \\
\hline
\end{tabular}

El tamaño de los campos es muy heterogéneo, mientras que algunos están constituidos por una única categoría, otros incluyen más de 50. Los mayores impactos corresponden a C15 (9.747, Multidisciplinary), C14 (5.083, Molecular Biology \& Genetics) y C22 (4.621, Space Science). Los menores impactos se alcanzan en C12 (1.345, Mathematics), C4 (1.529, Computer Science) y C6 (1.642, Economics \& Business).

Los campos de mayor crecimiento son C21 (0.63, Social Sciences, general) y C6 (0.62, Economics \& Business). Esto se debe a la importante incorporación de revistas en algunas de sus categorías en los últimos años. El único que reduce su tamaño, con un ratio por debajo del 0.5, es C22 (0.47, Space Science).

Las mayores referencias promedio se producen en C22 (56.59, Space Science) y C14 (51.64, Molecular Biology \& Genetics). Los menores promedios se obtienen en C12 (25.75, Mathematics) y C7 (27.74, Engineering). La mayor proporción de referencias JCR se alcanza en C14 (0.92, Molecular Biology \& Genetics) y las menores en C21 (0.61, Social Sciences, general), C4 (0.63, Computer Science) y C6 (0.66, Economics \& Business). La mayor proporción de ítems del JCR en la ventana de citación se alcanza en C22 (0.24, Space Science), y la menor en C19 (0.14, Plant \& Animal Science). Los mayores ratios entre ítems citados y citantes se obtienen en C15 (2.55, Multidisciplinary) y C14 (1.06, Molecular Biology \& Genetics). Los menores ratios corresponden a C21 (0.49, Social Sciences, general) y C6 (0.59, Economics \& Business).

Un Análisis Clúster ha determinado que C15 (Multidisciplinary) presenta componentes significativamente diferentes al resto, por lo que no puede ser agrupado junto a ningún otro campo. Los campos C6 (Economics \& Business) y C21 (Social Sciences, general) comparten un primer clúster mientras que los restantes 19 campos estarían asignados a un segundo clúster.

La Tabla 3 y la Figura 1 muestran las componentes para las áreas científicas. El área con mayor impacto agregado es Life Sciences \& Biomedicine. La 
componente más determinante sobre este valor es el número de referencias promedio. Las Social Sciences tienen el menor impacto agregado a pesar de contar con los mayores crecimientos y referencias promedio. Su bajo impacto viene justificado por la pequeña proporción de referencias del JCR y el ratio entre ítems citados y citantes. Technology tiene el segundo impacto agregado más pequeño a pesar de contar con la mayor proporción de ítems del JCR en la ventana de citación.

Tabla 3. Factor de impacto agregado y componentes para las áreas científicas de las bases de datos de Thomson Reuters.

\begin{tabular}{|l|l|r|r|r|r|r|c|}
\hline \multicolumn{1}{|c|}{ Área } & \multicolumn{1}{c|}{ Campos } & \multicolumn{6}{c|}{ Componentes del FIA } \\
\cline { 3 - 8 } & & $a_{t}^{F}$ & $r_{t}^{F}$ & $p_{t}^{F}$ & $w_{t}^{F}$ & $b_{t}^{F}$ & $F_{t}^{F}$ \\
\hline $\begin{array}{l}\text { Life Sciences } \\
\text { \& Biomedicine }\end{array}$ & $\begin{array}{l}\text { C1, C2, C3, C8, } \\
\text { C10, C13, C14, C16, } \\
\text { C17, C19 }\end{array}$ & 0.55 & 42.80 & 0.87 & 0.18 & 0.90 & 3.391 \\
\hline $\begin{array}{l}\text { Physical } \\
\text { Sciences }\end{array}$ & C5, C9, C12, C18, C22 & 0.54 & 34.97 & 0.87 & 0.18 & 0.88 & 2.667 \\
\hline Technology & $\mathrm{C4}, \mathrm{C7}, \mathrm{C} 11$ & 0.55 & 28.66 & 0.78 & 0.20 & 0.85 & 2.058 \\
\hline Social Sciences & $\mathrm{C6}, \mathrm{C} 20, \mathrm{C} 21$ & 0.60 & 46.15 & 0.67 & 0.18 & 0.60 & 2.001 \\
\hline
\end{tabular}

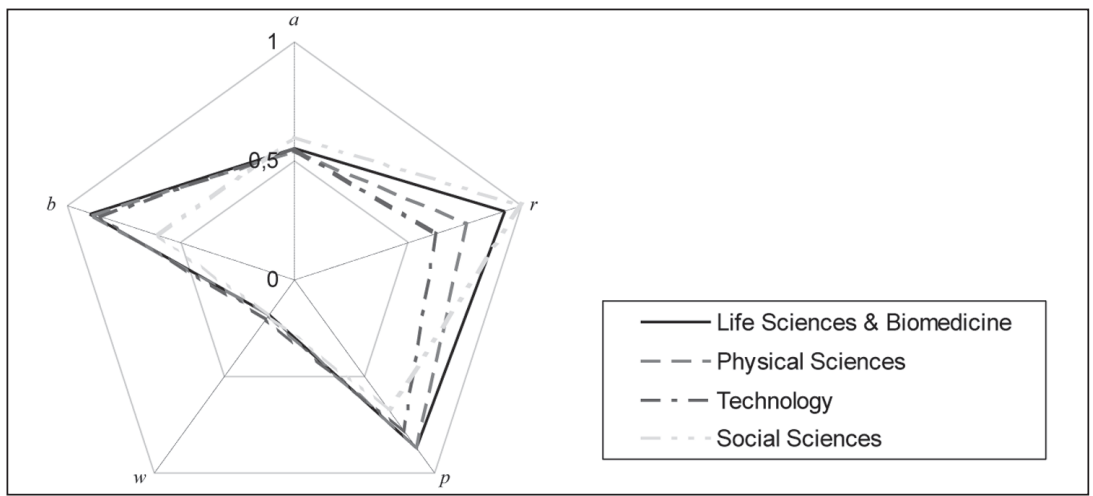

Figura 1. Componentes del factor de impacto agregado de las áreas Thomson Reuters.

El Anexo 1 muestra las categorías de revistas del JCR que constituyen cada campo junto al impacto agregado de la categoría y su porcentaje dentro de todo el campo. El impacto en Ciencias es un 58 \% mayor que en Ciencias Sociales. Esto se debe a que, a pesar de que en promedio existe un $30 \%$ más de referencias en los artículos de Ciencias Sociales, una parte importante de éstas son ítems no incluidos en el JCR. En concreto, un $40 \%$ de las referencias en Ciencias Sociales son libros y revistas no indexadas en el JCR, mientras que en Ciencias este porcentaje es sólo del $20 \%$. 
La varianza del impacto es grande dentro de cada edición. En Ciencias, las categorías con mayor impacto están relacionadas con la biomedicina, mientras que los menores impactos se obtienen en ingeniería y matemáticas. Con respecto a Ciencias Sociales, las categorías con mayor impacto corresponden a psicología y economía, mientras que los menores impactos se obtienen en categorías relacionadas con la historia.

Un Análisis de Componentes Principales ha determinado que la mayor parte de la varianza del impacto en Ciencias se explica con tres componentes principales: la proporción de referencia del JCR, la proporción de referencias del JCR en la ventana de citación y el crecimiento del propio campo. Por otro lado, en Ciencias Sociales la mayor parte de la varianza se explica con sólo dos componentes principales: la proporción de referencias del JCR en la ventana de citación y el ratio entre ítems citados y citantes. Las componentes principales son diferentes dependiendo de la edición del JCR. Esto es debido a que las Ciencias Sociales incluyen disciplinas tan heterogéneas en sus hábitos de publicación y citación como la economía, la psicología y la historia, por ejemplo.

Un Análisis Clúster ha identificado en primer lugar dos grupos de categorías de revistas que incluyen, en general, las ciencias de la vida que tienen un componente social más importante, y aquellas ciencias sociales que usan en mayor medida métodos matemáticos (psicología, economía y empresa). Sin embargo, existen importantes diferencias entre estos dos grupos. Un tercer grupo contiene aquellas áreas de ciencias sociales que usan en menor medida los métodos matemáticos (educación, sociología, lengua y derecho). Finalmente, un cuarto grupo incluye a las ciencias físicas y las de la vida (matemáticas, física, química, ingeniería y biomedicina).

\section{Conclusiones}

Los indicadores de impacto de las revistas no son comparables entre campos científicos debido a diferencias sistemáticas en los hábitos de publicación y citación. El objetivo de este trabajo es identificar las fuentes de varianza basándose en campos científicos. La identificación de dichas fuentes es fundamental a la hora de llevar a la práctica los indicadores bibliométricos normalizados y ajustados a las particularidades de cada campo.

Se presenta aquí una descomposición del factor de impacto en cinco fuentes principales de varianza. Además de las variables identificadas en la literatura (el número de referencias promedio y la proporción de las referencias del JCR), en este trabajo se constatan tres nuevas fuentes de varianza: el 
índice de crecimiento del campo, la proporción de referencias del JCR en la ventana de citación y la proporción entre ítems citados y citantes. Por tanto, es necesario considerar también estas nuevas variables cuando se quieren normalizar los factores de impacto y no sólo las dos variables que se han propuesto en la literatura.

\section{REFERENCIAS}

Althouse, B. M.; West, J. D.; Bergstrom, C. T. y Bergstrom, T. (2009), "Differences in impact factor across fields and over time", en Journal of the American Society for Information Science and Technology, 60 (1), pp. 27-34.

Bensman, S. J. (2007), "Garfield and the impact factor", en Annual Review of Information Science and Technology, 41 (1), pp. 93-155.

Bornmann, L. y Daniel, H. D. (2008), "What do citation counts measure? A review of studies on citing behavior", en Journal of Documentation, 64 (1), pp. 45-80.

Dorta-González, P. y Dorta-González, M. I. (2010), "Indicador bibliométrico basado en el índice h", en Revista Española de Documentación Científica, 33 (2), pp. 225-245.

_- (2011a), "Aplicación empírica de un indicador bibliométrico basado en el índice h", en Cultura y Educación, 23 (2), pp. 297-313.

- (2011b), "Central indexes to the citation distribution: A complement to the h-index", en Scientometrics, 88 (3), pp. 729-745.

_ (2013a), "Comparing journals from different fields of science and social science through a JCR subject categories normalized impact factor", en Scientometrics, 95 (2), pp. 645-672.

- (2013b), "Impact maturity times and citation time windows: The 2-year maximum journal impact factor", en Journal of Informetrics, 7 (3), pp. 593-602.

Egghe, L. y Rousseau, R. (2002), "A general framework for relative impact indicators", en Canadian Journal of Information and Library Science, 27 (1), pp. 29-48.

Garfield, E. (1972), "Citation analysis as a tool in journal evaluation", en Science, 178 (4060), pp. 471-479.

(1979a), Citation indexing: Its theory and application in Science, Technology, and Humanities, New York, John Wiley.

_ (1979b), "Is citation analysis a legitimate evaluation tool?", en Scientometrics, 1 (4), pp. 359-375.

Leydesdorff, L. (2006), "Can scientific journals be classified in terms of aggregated journal-journal citation relations using the Journal Citation Reports?", en Journal of the American Society for Information Science \& Technology, 57 (5), pp. 601-613. 
— y Bornmann, L. (2011), "How fractional counting of citations affects the Impact Factor: Normalization in terms of differences in citation potentials among fields of science", en Journal of the American Society for Information Science E Technology, 62 (2), pp. 217-229.

— y Opthof, T. (2010), "Normalization at the field level: Fractional counting of citations", en Journal of Informetrics, 4 (4), pp. 644646.

y Rafols, I. (2011), "Indicators of the interdisciplinarity of journals: Diversity, centrality, and citations", en Journal of Informetrics, 5 (1), pp. 87-100.

Moed, H. F. (2010), "Measuring contextual citation impact of scientific journals”, en Journal of Informetrics, 4 (3), pp. 265-277.

Opthof, T. y Leydesdorff, L. (2010), "Caveats for the journal and field normalizations in the CWTS ('Leiden') evaluations of research performance", en Journal of Informetrics, 4 (3), pp. 423-430.

Pudovkin, A. I. y Garfield, E. (2002), "Algorithmic procedure for finding semantically related journals", en Journal of the American Society for Information Science and Technology, 53 (13), pp. 11131119.

Rafols, I. y Leydesdorff, L. (2009), “Content-based and algorithmic classifications of journals: Perspectives on the dynamics of scientific communication and indexer effects", en Journal of the American Society for Information Science and Technology, 60 (9), pp. 1823-1835.

Rosvall, M. y Bergstrom, C. T. (2008), "Maps of random walks on complex networks reveal community structure", en Proceedings of the National Academy of Sciences, 105 (4), pp. 1118-1123.

Van Raan, A. F. J.; Van Leeuwen, T. N.; Visser, M. S.; Van Eck, N. J. y Waltman, L. (2010), "Rivals for the crown: Reply to Opthof and Leydesdorff”, en Journal of Informetrics, 4 (3), pp. 431-435.

Wagner, C.; Roessner, J. D.; Bobb, K.; Klein, J.; Boyack, K.; Keyton, J.; Rafols, I. y Börner, K. (2011), "Approaches to understanding and measuring interdisciplinary scientific research (IDR): A review of the literature", en Journal of Informetrics, 5 (1), pp. 14-26.

Zitt, M. y Small, H. (2008), "Modifying the journal impact factor by fractional citation weighting: The audience factor", en Journal of the American Society for Information Science and Technology, 59 (11), pp. 1856-1860. 
Campos científicos, categorías de revistas $J C R$, porcentajes dentro del campo e impactos

1. Agricultural Sciences: Agricultural Engineering, 6\%, 3.123; Agriculture, Multidisciplinary, 13\%, 1.673; Agronomy, $15 \%, 1.774$; Food Science \& Technology, $40 \%, 1.942$; Horticulture, $7 \%, 1.429$; Nutrition \& Dietetics, $19 \%, 3.098$.

2. Biology \& Biochemistry: Anatomy \& Morphology, $1 \%$, 1.976; Biochemical Research Methods, 9 \%, 3.822; Biochemistry \& Molecular Biology, 32\%, 4.435; Biology, $10 \%$, 4.114; Biophysics, 8 \%, 3.291; Biotechnology \& Applied Microbiology, 15 \%, 3.256; Endocrinology \& Metabolism, 9\%, 4.304; Evolutionary Biology, $3 \%, 4.116$; Mathematical \& Computational Biology, 3\%, 3.038; Microscopy, 1\%, 2.293; Parasitology, $3 \%, 3.056$; Physiology, $6 \%, 3.223$.

3. Clinical Medicine: Allergy, 0\%, 3.844; Andrology, 0\%, 2.377; Anesthesiology, 1\%, 2.955; Cardiac \& Cardiovascular Systems, 4\%, 4.277; Clinical Neurology, 5 \%, 3.238; Critical Care Medicine, $1 \%$, 3.924; Dentistry, Oral Surgery \& Medicine, 2\%, 1.966; Dermatology, 1\%, 2.525; Emergency Medicine, $1 \%$, 2.123; Endocrinology \& Metabolism, $3 \%, 4.304$; Engineering, Biomedical, $2 \%, 2.848$; Gastroenterology \& Hepatology, 2\%, 3.801; Geriatrics \& Gerontology, 1\%, 3.158; Health Care Sciences \& Services, $1 \%$, 2.154; Hematology, 2\%, 5.310; Immunology, 4\%, 4.585; Infectious Diseases, $2 \%, 3.879$; Integrative \& Complementary Medicine, 0 \%, 2.402; Materials Science, Biomaterials, 1\%, 3.729; Medical Informatics, 0 \%, 1.893; Medical Laboratory Technology, 1\%, 2.208; Medicine, General \& Internal, 4\%, 4.754; Medicine, Legal, $0 \%, 1.787$; Medicine, Research \& Experimental, $3 \%, 3.753$; Neuroimaging, $0 \%, 4.098$; Nutrition \& Dietetics, 2\%, 3.098; Obstetrics \& Gynecology, $2 \%, 2.397$; Oncology, $6 \%$, 4.941; Ophthalmology, $2 \%$, 2.379; Orthopedics, $2 \%$, 2.048; Otorhinolaryngology, 1\%, 1.501; Pathology, $2 \%, 2.763$; Pediatrics, $3 \%$, 2.005; Peripheral Vascular Disease, $2 \%$, 4.612; Pharmacology \& Pharmacy, 7 \% , 3.134; Physiology, 2\%, 3.223; Primary Health Care, 0\%, N.A.; Public, Environmental \& Occupational Health (Science), $3 \%, 2.666$; Public, Environmental \& Occupational Health (Social Science), 2\%, 2.177; Radiology, Nuclear Medicine \& Medical Imaging, 4 \% , 2.972; Rehabilitation, $1 \%$, 2.103; Rehabilitation, 1 \%, 1.632; Reproductive Biology, 1\%, 2.904; Respiratory System, 2\%, 3.475; Rheumatology, 1\%, 4.133; Sport Sciences, 2\%, 2.300; Surgery, 7\%, 2.272; Transplantation, 1\%, 2.876; Tropical Medicine, 1\%, 2.400; Urology \& Nephrology, $2 \%, 3.078$.

4. Computer Science: Computer Science, Artificial Intelligence, 16\%, 1.940; Computer Science, Cybernetics, $2 \%, 1.395$; Computer Science, Hardware \& Architecture, $7 \%$, 1.203; Computer Science, Information Systems, $15 \%$, 1.583; Computer Science, Interdisciplinary Applications, 18 \%, 1.652; Computer Science, Software Engineering, $12 \%$, 1.240; Computer Science, Theory \& Methods, $10 \%, 1.404$; Imaging Science \& Photographic Technology, $3 \%$, 2.186; Telecommunications, $17 \%, 1.331$.

5. Chemistry: Biochemical Research Methods, $6 \%, 3.822$; Crystallography, $4 \%, 1.681$; Chemistry, Analytical, $7 \%$, 2.906; Chemistry, Applied, $5 \%$, 2.207; Chemistry, Inorganic \& Nuclear, $5 \%, 2.404$; Chemistry, Medicinal, $5 \%$, 2.795; Chemistry, Multidisciplinary, $16 \%$, 4.586; Chemistry, Organic, $8 \%, 2.853$; Chemistry, Physical, 17 \%, 3.615; Electrochemistry, 4\%, 3.615; Engineering, Chemical, $9 \%, 1.940$; Materials Science, Textiles, 1\%, 1.208; Physics, Atomic, Molecular \& Chemical, 6 \%, 2.344; Polymer Science, $6 \%, 2.508$; Spectroscopy, $2 \%, 2.065$.

6. Economics \& Business: Agricultural Economics \& Policy, 1\%, 1.088; Business, $14 \%, 1.845$; Business, Finance, $10 \%, 1.602$; Economics, $46 \%, 1.459$; History of Social Sciences, $2 \%, 0.623$; Industrial Relations \& Labor, $2 \%, 1.208$; Management, $18 \%$, 2.249; Social Sciences, Mathematical Methods, 6 \%, 1.392.

7. Engineering: Automation \& Control Systems, $2 \%, 1.532$; Computer Science, Artificial Intelligence, $2 \%$, 1.940; Computer Science, Cybernetics, 0\%, 1.395; Computer Science, Hardware \& Architecture, $1 \%$, 1.203; Computer Science, Interdisciplinary Applications, $3 \%$, 1.652; Construction \& Building Technology, 1\%, 1.121; Energy \& Fuels, 4 \%, 2.912; Engineering, Aerospace, $1 \%, 0.628$; Engineering, Biomedical, $3 \%, 2.848$; Engineering, Civil, $3 \%, 1.593$; Engineering, Chemical, $6 \%, 1.940$; Engineering, Electrical \& Electronic, 11\%, 1.541; Engineering, Environmental, 3\%, 3.258; Engineering, Geological, 1\%, 1.132; Engineering, Industrial, 1\%, 1.450; Engineering, Manufacturing, 1\%, 1.307; Engineering, Marine, $0 \%$, 0.207; Engineering, Mechanical, 3\%, 1.127; Engineering, Multidisciplinary, 2\%, 0.928; Engineering, 
FACTOR DE IMPACTO AGREGADO SEGÚN CAMPOS CIENTÍFICOS

Ocean, $0 \%, 0.998$; Engineering, Petroleum, $0 \%, 0.565$; Ergonomics, $0 \%, 1.436$; Instruments \& Instrumentation, $3 \%, 1.675$; Materials Science, Characterization \& Testing, $1 \%$, 0.939; Mathematics, Applied, $6 \%, 1.247$; Mathematics, Interdisciplinary Applications, $2 \%, 1.515$; Mechanics, $4 \%$, 1.574; Nanoscience \& Nanotechnology, $6 \%$, 4.365; Nuclear Science \& Technology, $2 \%, 1.025$; Operations Research \& Management Science, 2 \%, 1.557; Physics, Applied, 12 \%, 2.724; Physics, Fluids \& Plasmas, $2 \%, 2.151$; Remote Sensing, 1\%, 1.948; Robotics, $0 \%$, 1.795; Spectroscopy, $2 \%$, 2.065; Telecommunications, $3 \%$, 1.331; Thermodynamics, $2 \%, 1.608$; Transportation Science \& Technology, 1\%, 0.957; Water Resources, $3 \%, 1.764$.

8. Environment/Ecology: Biodiversity Conservation, 4\%, 2.688; Ecology, 20 \%, 3.094; Engineering, Environmental, $13 \%$, 3.258; Environmental Sciences, 37 \%, 2.507; Environmental Studies, $6 \%$, 2.027; Geography, Physical, 5 \%, 2.323; Limnology, $3 \%, 2.028$; Water Resources, $13 \%, 1.764$.

9. Geosciences: Energy \& Fuels, 20 \% , 2.912; Engineering, Geological, $3 \%, 1.132$; Engineering, Petroleum, $2 \%, 0.565$; Geochemistry \& Geophysics, $11 \%, 2.358$; Geography, Physical, $5 \%, 2.323$; Geology, $3 \%$, 1.868; Geosciences, Multidisciplinary, $25 \%$, 2.230; Meteorology \& Atmospheric Sciences, 12\%, 2.475; Mineralogy, $3 \%, 1.790$; Mining \& Mineral Processing, $3 \%, 1.033$; Oceanography, $7 \%, 1.943$; Paleontology, $3 \%, 1.873$; Remote Sensing, $3 \%, 1.948$

10. Immunology: Immunology, 68 \%, 4.585; Infectious Diseases, $32 \%, 3.879$.

11. Materials Science: Construction \& Building Technology, $3 \%, 1.121$; Materials Science, Biomaterials, $4 \%$, 3.729; Materials Science, Ceramics, 3\%, 1.264; Materials Science, Coatings \& Films, $5 \%$, 1.943; Materials Science, Composites, $2 \%, 1.553$; Materials Science, Characterization \& Testing, $2 \%, 0.939$; Materials Science, Multidisciplinary, $48 \%$, 2.949; Materials Science, Paper \& Wood, 1\%, 0.912; Materials Science, Textiles, 1\%, 1.208; Metallurgy \& Metallurgical Engineering, 12 \%, 1.346; Nanoscience \& Nanotechnology, $18 \%, 4.365$.

12. Mathematics: Mathematical \& Computational Biology, $7 \%$, 3.038; Mathematics, $29 \%$, 0.829; Mathematics, Applied, $30 \%$, 1.247; Mathematics, Interdisciplinary Applications, $10 \%$, 1.515; Physics, Mathematical; $14 \%, 1.726$; Statistics \& Probability, $10 \%, 1.241$.

13. Microbiology: Microbiology, 56 \%, 3.801; Microscopy, 3 \%, 2.293; Mycology, 5 \%, 2.059; Parasitology, $14 \%, 3.056$; Virology, $21 \%, 4.122$

14. Molecular Biology \& Genetics: Biochemistry \& Molecular Biology, $53 \%$, 4.435; Cell \& Tissue Engineering, 1\%, N.A.; Cell Biology, $24 \%$, 6.453; Developmental Biology, 4\%, 4.583; Genetics \& Heredity, $18 \%$, 4.861.

15. Multidisciplinary: Multidisciplinary Sciences, $100 \%, 9.707$.

16. Neuroscience \& Behavior: Behavioral Sciences, $8 \%, 3.048$; Clinical Neurology, $36 \%, 3.238$; Neuroimaging, $3 \%, 4.098$; Neurosciences, $50 \%, 4.082$; Psychology, Biological, $2 \%, 2.682$.

17. Pharmacology \& Toxicology: Chemistry, Medicinal, 23 \%, 2.795; Pharmacology \& Pharmacy, 59 \%, 3.134; Toxicology, $18 \%, 2.765$.

18. Physics: Acoustics, 2\%, 1.553; Imaging Science \& Photographic Technology, 1\%, 2.186; Optics, $13 \%$, 2.204; Physics, Applied, 25 \%, 2.724; Physics, Atomic, Molecular \& Chemical, 9 \%, 2.344; Physics, Condensed Matter, $16 \%$, 3.095; Physics, Fluids \& Plasmas, 5 \%, 2.151; Physics, Mathematical, $6 \%$, 1.726; Physics, Multidisciplinary, $13 \%$, 3.046; Physics, Nuclear, $3 \%$, 1.796; Physics, Particles \& Fields, $6 \%$, 3.503.

19. Plant \& Animal Science: Agriculture, Dairy \& Animal Science, 7\%, 1.428; Entomology, $6 \%, 1.409$; Evolutionary Biology, $6 \%$, 4.116; Fisheries, $5 \%$, 1.579; Forestry, $4 \%$, 1.607; Horticulture, $3 \%, 1.429$; Limnology, $2 \%, 2.028$; Marine \& Freshwater Biology, $10 \%, 1.870$; Mycology, $2 \%, 2.059$; Oceanography, $5 \%, 1.943$; Ornithology, 1\%, 1.182; Plant Sciences, 19\%, 2.692; Reproductive Biology, 5\%, 2.904; Veterinary Sciences, $15 \%, 1.213$; Zoology, $11 \%, 1.613$.

20. Psychiatry/Psychology: Behavioral Sciences, 8\%, 3.048; Criminology \& Penology, 2\%, 1.260; Ergonomics, $2 \%$, 1.436; Family Studies, 3\%, 1.449; Psychiatry, $19 \%$, 3.507; Psychiatry, 12\%, 3.215; Psychology, $8 \%$, 2.741; Psychology, Applied, 4\%, 1.812; Psychology, Biological, 2\%, 2.682; Psychology, Clinical, 9 \%, 2.459; Psychology, Developmental, 6 \%, 2.572; Psychology, Educational, $3 \%, 1.637$; Psychology, Experimental, 9 \%, 2.590; Psychology, Mathematical, 1\%, 1.840; Psychology, Multidisciplinary, 9 \%, 2.098; Psychology, Psychoanalysis, 1\%, 1.147; Psychology, Social, 5 \%, 1.835. 
21. Social Sciences, general: Anthropology, 2\%, 1.381; Area Studies, 1\%, 0.640; Communication, 1\%, 1.271; Criminology \& Penology, 1\%, 1.260; Cultural Studies, 0 \%, N.A.; Demography, $1 \%, 1.258$; Education \& Educational Research, $5 \%$, 1.242; Education, Scientific Disciplines, $2 \%, 1.529$; Education, Special, 1\%, 1.574; Environmental Studies, 3\%, 2.027; Ethics, 1\%, 1.232; Ethnic Studies, 0 \%, 1.203; Family Studies, 1\%, 1.449; Geography, $2 \%, 1.644$; Geriatrics \& Gerontology, $3 \%$, 3.158; Gerontology, $1 \%$, 2.335; Health Care Sciences \& Services, 4 \%, 2.154; Health Policy \& Services, $3 \%, 2.271$; History, $1 \%$, 0.479; History \& Philosophy of Science (Science), $1 \%, 0.754$; History \& Philosophy of Science (Social Science), $1 \%, 0.922$; History of Social Sciences, $1 \%, 0.623$; Hospitality, Leisure, Sport \& Tourism, $1 \%$, 2.212; Industrial Relations \& Labor, $0 \%, 1.208$; Information Science \& Library Science, $2 \%, 1.430$; International Relations, 2\%, 1.078; Law, $3 \%, 1.495$; Linguistics, $2 \%, 1.471$; Medical Ethics, $0 \%, 1.581$; Medicine, Legal, 1\%, 1.787; Nursing (Science), 4\%, 1.369; Nursing (Social Science), 4\%, 1.367; Planning \& Development, $2 \%, 1.233$; Political Science, 4\%, 1.011; Psychology, Educational, 1\%, 1.637; Public Administration, 1\%, 1.199; Public, Environmental \& Occupational Health (Science), $10 \%, 2.666$; Public, Environmental \& Occupational Health (Social Science), $6 \%, 2.177$; Rehabilitation (Science), $2 \%$, 2.103; Rehabilitation, $2 \%, 1.632$; Social Issues (Science), $3 \%, 1.721$; Social Issues (Social Science), 1\%, 1.043; Social Sciences, Biomedical, $2 \%, 2.002$; Social Sciences, Interdisciplinary, $3 \%, 1.227$; Social Work, 1\%, 1.201; Sociology, 3\%, 1.111; Substance Abuse (Science), 1\%, 2.959; Substance Abuse (Social Science), 1\%, 2.261; Transportation, 1\%, 1.874; Urban Studies, 1\%, 1.211; Women's Studies, $1 \%, 1.048$.

22. Space Science: Astronomy \& Astrophysics, $100 \%, 4.609$. 